\title{
La rénovation énergétique des bâtiments en France entre marché, actions territoriales et dispositions nationales
}

Retour d'expérience des OPATB de l'agglomération grenobloise

\section{Gilles Debizet}

\section{OpenEdition}

\section{Journals}

Édition électronique

URL : http://journals.openedition.org/developpementdurable/8856

DOI : 10.4000/developpementdurable.8856

ISSN : 1772-9971

Éditeur

Association DD\&T

Référence électronique

Gilles Debizet, «La rénovation énergétique des bâtiments en France entre marché, actions territoriales et dispositions nationales ", Développement durable et territoires [En ligne], Vol. 2, n 1 | Mars 2011, mis en ligne le 21 janvier 2013, consulté le 10 décembre 2020. URL : http://journals.openedition.org/ developpementdurable/8856 ; DOI : https://doi.org/10.4000/developpementdurable.8856

Ce document a été généré automatiquement le 10 décembre 2020.

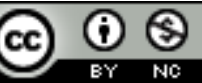

Développement Durable et Territoires est mis à disposition selon les termes de la licence Creative Commons Attribution - Pas d'Utilisation Commerciale 4.0 International. 


\title{
La rénovation énergétique des bâtiments en France entre marché, actions territoriales et dispositions nationales
}

Retour d'expérience des OPATB de l'agglomération grenobloise

\author{
Gilles Debizet
}

Le bâtiment résidentiel et tertiaire représente $43 \%$ de la consommation finale d'énergie et émet $23 \%$ des émissions de gaz à effet de serre (GES) en France. Moins émetteur que les transports ou l'industrie, ce secteur apparaît cependant comme le principal gisement de réduction des émissions (CGDD, 2009). Depuis 1975, la construction s'est adaptée aux renforcements successifs de la réglementation thermique. Le chauffage des bâtiments récents les plus performants consomme 10 fois moins d'énergie que celui des bâtiments existants. Compte tenu de la très faible part de bâtiments anciens détruits, le facteur 4 à l'horizon 2050 est plausible dans ce secteur si l'essentiel des bâtiments existants à ce jour est rénové.

Parmi les «chantiers" thématiques du Grenelle de l'Environnement, le «bâtiment ancien » fut l'un des plus gros contributeurs à la réduction envisagée des émissions de GES d'ici 2050 et celui pour lequel les parties impliquées convergèrent facilement sur des objectifs à moyen terme. D'une certaine façon, l'attention des acteurs du Grenelle s'est portée sur la rénovation énergétique avec le même enthousiasme que pour le neuf : la loi Grenelle vise une réduction de $38 \%$ de la consommation d'énergie primaire dès 2020 . L'habitat représente la majorité de la surface bâtie, la propriété de ces bâtiments est essentiellement assurée par des particuliers ${ }^{1}$. La disjonction (Berdoulay, 2002) entre l'échelle du problème (le réchauffement climatique planétaire) et l'échelle de décision (chaque propriétaire de logement) ne peut être plus grande. La solidarité des propriétaires vis-à-vis de la planète ne saurait suffire à atteindre le facteur 4 en France ; la rénovation énergétique exige l'action des pouvoirs publics. Pour les prochaines années, les travaux nécessaires ont été chiffrés à plusieurs centaines de milliards d'euros. 
L'État peine à rendre la rénovation énergétique obligatoire. Les voies du marché ont été explorées et mises en œuvre au niveau national: déductions fiscales, certificats d'économie d'énergie, prêts bonifiés... Elles s'avèrent insuffisantes puisque l'État et des collectivités locales complètent ces facilités par des subventions substantielles. Se pose la question des conditions d'attribution de l'argent public à des propriétaires privés. Le compromis entre l'efficacité environnementale, l'effet de levier économique et l'équité sociale pourrait varier selon les territoires.

Le présent article se propose d'explorer un nouveau dispositif élaboré et mise en œuvre dans la première décennie du XXI ${ }^{e}$ siècle, centré sur les économies d'énergie et la rédaction des émissions de gaz à effet de serre : l'Opération Programmée d'amélioration énergétique et thermique des bâtiments (OPATB). Ce dispositif national et les opérations engendrées engagent une grande variété d'acteurs décisionnels et de prestataires dans des projets innovants et incertains tant en termes de résultats que de méthodes. Pour le décrire, nous choisissons d'utiliser le concept d'objet-frontière (Star, 1989; Trompette, 2009). Ce concept permet de définir les processus par lesquels des acteurs de mondes sociaux différents réussissent à se coordonner malgré leur point de vue différents. Simultanément à l'analyse des interactions (plus ou moins développée dans le présent article selon les sources disponibles), il s'intéresse aux infrastructures de connaissances telles que les conventions et normes attachées à une communauté de pratiques. Dans le cas de l'action publique, nous supposerons que ces dernières sont liées à des procédures propres (ou partiellement partagées par) aux différentes organisations impliquées.

Dans la première partie, le dispositif OPATB est analysé en tant que procédure nationale rassemblant les organisations étatiques en charge de l'habitat et de l'environnement. Basée sur des documents officiels, cette partie aborde cet objet-frontière à partir des infrastructures organisationnelles. La deuxième partie considère l'OPATB comme un projet multiterritorial et relate la genèse des OPATB des communes voisines de Grenoble et d'Echirolles, Elle met en évidence les dimensions territoriales d'un dispositif trop souvent cantonné à sa technicité, articulant ainsi les dimensions urbaine et sociotechnique ${ }^{2}$. La troisième partie se focalise sur les résultats et les apprentissages de l'OPATB de Grenoble. Outre des documents de projets, sont mobilisées dans cette troisième partie des travaux d'étudiants en master Sciences du Territoire menés en partenariat avec des acteurs de l'OPATB ainsi que des échanges de l'auteur avec les techniciens et des élus locaux. Enfin, la quatrième illustre les apprentissages et les mutations envisagées en présentant le dispositif intercommunal qui prend la suite des OPATB de Grenoble et Echirolles.

\section{Le dispositif national OPATB, une construction hybride au confluent de politiques socioéconomiques et climatiques}

Le bâtiment intéresse depuis longtemps le ministère du Logement et celui de l'Économie et des Finances. Le changement climatique a réintroduit un acteur devenu très influent: le ministère de l'Environnement («Écologie» depuis 2007). Ces acteurs, ainsi que les agences nationales "opératrices" des deux derniers ont été à l'origine du dispositif incitatif OPATB. 


\subsection{De la difficulté de fixer une obligation de rénover les bâtiments}

Lors du Grenelle de l'environnement, les associations environnementalistes ont pesé pour rendre obligatoire la rénovation énergétique. Le comité opérationnel «rénovation des bâtiments existants" s'est montré réticent argumentant sur la solvabilité des propriétaires et la difficulté à définir des obligations contrôlables (Pelletier, 2008). Malgré le consensus général sur l'amélioration énergétique des bâtiments existants, les lois dites « Grenelle 1 » et « Grenelle 2 » ne n'imposent pas d'obligation généralisée.

Depuis son instauration en 1975, le renforcement de la réglementation thermique des bâtiments ne concerne que les bâtiments soumis à une procédure d'autorisation de construire, condition nécessaire à la vérification de la conformité par une autorité publique. D'une façon générale, le législateur et les services de l'État ne fixent des obligations que s'il existe des moyens de contrôle et de sanction. Ce contrôle peut être exercé directement par une autorité publique: la commune en dispose pour le ravalement des façades donnant sur le domaine public et pour les grosses réhabilitations soumises à une procédure d'autorisation ${ }^{3}$. Il peut aussi être aussi assis sur une transaction : le diagnostic de performance énergétique (DPE) est associé à la vente ou la location d'un logement. Enfin, des performances minimales sont imposées aux professionnels lorsqu'ils remplacent les ouvrants d'un bâtiment. Il n'en reste pas moins qu'aucune obligation thermique ne pèse sur un propriétaire immobilier qui n'entreprendrait pas de travaux.

Finalement, ce sont des dispositions incitatives qui ont été - et probablement seront promues pendant plusieurs années. Nombre d'entre elles ont été conçues dans le cadre de politiques nationales anciennes répondant à d'autres objectifs que la convergence vers le facteur 4 .

\subsection{Des systèmes préexistants d'aide financière aux propriétaires}

Avec 1,5 millions d'emplois, le secteur du bâtiment constitue l'un des premiers secteurs économiques du pays. À ce titre, le bâtiment neuf bénéficie depuis longtemps de soutien de grande ampleur: crédit d'impôt pour les investisseurs particuliers, déduction des intérêts d'emprunt pour les acquéreurs occupants, prêt à taux zéro, Pass Foncier. Les travaux d'amélioration bénéficient du taux réduit de TVA (5,5\% au lieu de 19,6\%) depuis 1999. Progressivement, des conditions plus avantageuses ont été consenties pour les travaux d'amélioration liés à l'efficacité énergétique des bâtiments ${ }^{4}$.

Les dispositions fiscales et les bonifications de prêts se sont toujours avérées insuffisantes pour stimuler l'investissement des propriétaires bailleurs et permettre celui des propriétaires occupants les plus modestes. Les premiers peinent à amortir leur investissement du fait du plafonnement des loyers. Les seconds manquent de trésorerie ou de capacité d'emprunt pour investir dans des travaux.

Ces deux catégories bénéficient d'un soutien prioritaire de l'Agence Nationale pour l'Habitat. Créée par l'État en 1971 pour promouvoir le confort et l'entretien de l'habitat privé, l'Anah "encourage et facilite l'exécution de travaux d'amélioration et d'adaptation de l'habitat» (Code de la construction et de l'habitation article L321-1). Cette agence finance de façon croissante le développement du parc privé en attribuant directement des subventions pour travaux aux ménages appartenant à ces deux catégories. 
Les partenariats avec les collectivités locales ont pris une place croissante dans l'activité de l'Anah. Les premières Opérations programmées d'amélioration de l'habitat (OPAH) ont été signées avec des communes en 1977, dans le cadre de rénovation urbaine de quartiers historiques délaissés (Gravejat, 1991). Progressivement, l'Anah a étendu leur champ d'application pour répondre aux attentes des collectivités locales ${ }^{5}$. In fine en 2008, 2/3 des 526 millions d'euros de subventions distribuées par l'Anah ont été accordées dans le cadre d'opérations programmées, au nombre de 670 réparties sur le territoire national ${ }^{6}$. Un volet thermique est introduit au cours des années 1990. Il prend de l'ampleur à tel point que, 273 des 715 opérations programmées en cours en avril 2009 intègrent la thématique «maitrise énergétique ».

L'octroi de subventions publiques à des personnes privées est justifié par l'effet de levier ${ }^{7}$. Lorsqu'elle concerne des immeubles dans des quartiers centraux à fort potentiel, l'amélioration de l'habitat peut conduire à une gentrification (Gravejat, 1991). Comme nous le verrons plus loin, ces problématiques conduisent les collectivités locales à une certaine prudence par rapport au financement public et induisent des montages complexes pour la rénovation énergétique des copropriétés.

À côté de l'Anah, l'Agence De l'Environnement et de la Maîtrise de l'Énergie (Ademe) finance des études et des travaux de rénovation énergétique au titre de sa mission de mise en œuvre des politiques environnementales de l'État, elle met aussi sa capacité d'expertise à disposition des collectivités locales. L'Ademe contractualise avec les Régions : une Région peut abonder des dispositifs nationaux pour offrir des subventions plus avantageuses aux propriétaires, elle peut aussi élaborer avec l'Ademe des dispositifs différents. Ces dispositifs sont généralement calibrés pour faire émerger des expériences innovantes ou amorcer une diffusion générale. Lorsque l'Ademe s'en retire, ils sont éventuellement pérennisés par des dispositions de droit commun de l'État ou les grandes collectivités locales.

\subsection{La genèse du dispositif national OPATB}

«Les OPATB ont pour objectif d'agir au niveau local sur les bâtiments résidentiels et tertiaires afin de limiter leurs consommations énergétiques et leurs émissions de $\mathrm{CO}_{2} \|^{8}$

Le dispositif OPATB a été présenté en février 2002 sous la forme d'un appel à projet associant l'Ademe, l'Anah et leur tutelle respective : ministère de l'Aménagement du Territoire et de l'Environnement et secrétariat d'État au Logement. Il s'inscrit respectivement dans les programmes d'actions ministériels, à savoir le Programme national de lutte contre le changement climatique (janvier 2000), le Programme national d'amélioration de l'efficacité énergétique (décembre 2000) et le Plan national habitat, construction et développement durable (janvier 2002).

En décembre 2000 lors de la réunion annuelle de l'Amélioration de l'habitat, le secrétaire d'État au Logement demande à l'Anah de s'impliquer dans les économies d'énergie sans en faire une nouvelle orientation". "... [J] rappellerai que les principaux gisements d'économie d'énergie se situent dans l'habitat existant. Je demande donc à l'Anah de s'impliquer dans la relance des économies d'énergies. Des dispositions devront être prises en ce sens en liaison avec l'Ademe, qui alimenteront les mesures que le Gouvernement s'apprête à annoncer dans ce domaine sous la responsabilité de Dominique Voynet [alors ministre de l'Aménagement du Territoire et de l'Environnement]» (Besson, 2000). Le secrétaire d'État appelle à un partenariat avec les collectivités locales sur ces thématiques techniques. Entre ce premier discours et le 
lancement officiel du dispositif OPATB, la sémantique du secrétariat d'État au Logement est passée d'une vision centrée sur le bâtiment « économies d'énergie » au concept multiscalaire de « développement durable » pour arriver à la solidarité planétaire de réduction des «émissions de $\mathrm{CO}_{2}$ ». Il fallut 14 mois concevoir et valider, au niveau interministériel, le dispositif.

Les OPATB s'inscrivent dans le moule des Opérations Programmées d'Amélioration de l'Habitat (OPAH). Concrètement, la collectivité délimite le territoire et fixe les objectifs, en concertation avec l'Anah. Les propriétaires privés bénéficient d'aides majorées par rapport à ce que l'Anah verse habituellement. L'Anah concentre ses moyens sur les propriétaires modestes et les propriétaires bailleurs. Les collectivités disposent de latitude pour définir les financements complémentaires à ces deux catégories ainsi qu'aux autres occupants, propriétaires ou locataires. Formellement, la spécificité de l'OPATB tient à la possibilité de financer des immeubles non-résidentiels en plus des logements ${ }^{10}$ et au ciblage exclusif des travaux sur la thématique énergie et $\mathrm{CO}_{2}$. Du fait de la diversité fonctionnelle des bâtiments ciblés, l'opération dépasse le secteur du logement, elle est potentiellement traitable, à l'échelle locale, comme un projet de quartier ou de territoire. L'Ademe cofinance des études préalables et opérationnelles, l'animation et l'évaluation et apporte un soutien technique et financier à la campagne de communication et aux programmes de formation. Il arrive qu'elle finance en plus des installations d'efficacité énergétique ou d'énergie renouvelable.

La phase opérationnelle d'une OPATB dure 4 ou 5 ans, elle est précédée d'études préopérationnelles qui permettent de préciser le périmètre et les types de travaux et de calibrer les niveaux de subvention et les enveloppes financières des partenaires qui s'engagent par convention pour toute la durée. Le taux de subvention doit être suffisamment élevé pour déclencher le réinvestissement des acteurs privés tout en étant limité par l'enveloppe allouée par chaque partenaire financeur. Cette tension se met en œuvre dans une grande incertitude puisqu'il revient toujours au propriétaire (ou au syndicat de copropriétaires) de décider in fine des travaux et, par conséquent, de mobiliser ou pas les subventions.

Avant le lancement de l'appel à projet auprès des collectivités locales en février 2002, 3 sites pilotes avaient été sélectionnés pour tester les modalités en grandeur nature, notamment le positionnement des collectivités locales. Il était envisagé de lancer 26 OPATB en 2002 et 2003 dans une perspective expérimentale: «De la réussite de ces premières OPATB dépend leur multiplication future $»^{11}$. Mais seules 16 OPATB étaient en cours en 2007.

Le dispositif national OPATB constitue une innovation organisationnelle, projet commun à plusieurs ministères associant deux agences de l'État aux finalités différentes. Le glissement sémantique des finalités (économies d'énergie/développement durable/ réduction des émissions de GES) révèle l'intégration des préoccupations climatiques par le ministère du logement. Sur le plan organisationnel, la procédure se base sur un dispositif existant de l'Anah qui a fait ses preuves en termes de partenariat avec les collectivités locales. Il est probable que l'Ademe ait apporté son expérience de la diffusion de l'innovation et son expertise en énergétique du bâtiment dès ce stade. Mais nos investigations n'ont pas porté sur cette question. C'est maintenant à travers la genèse des OPATB de Grenoble et Echirolles que nous analyserons la mise en œuvre du dispositif OPATB. 


\section{Les OPATB de Grenoble et Echirolles comme projets multi-scalaires}

De la commune à l'État, chaque partenaire inscrit l'OPATB dans un double objectif de réduction de la consommation d'énergie et des émissions de gaz à effet de serre, manifestant ainsi l'intention de lutter contre le réchauffement climatique. Ce double objectif apparaît tel quel dans les documents de communication grand public à Grenoble et à Echirolles. Cependant, chacun des acteurs rattache aussi l'OPATB à d'autres enjeux. Nous verrons que ces enjeux prédéterminent les modalités d'accompagnement et la répartition des rôles.

\subsection{Enjeux et logiques d'action pour l'État et ses agences nationales}

Évacuons d'abord l'État. Il n'est pas absent des choix: les ministères définissent les orientations des agences et le dispositif OPATB a été validé au niveau interministériel. Les ministères veillent ensuite au respect des orientations aux moments charnières (leurs représentants siègent dans le comité de sélection des collectivités) et aux retombées en termes de communication ${ }^{12}$. Bien-sûr les tensions persistent entre les objectifs environnementaux portés par le ministère de l'environnement/développement durable et les priorités sociales et économiques portées par le ministère du logement. Mais nous pouvons considérer, qu'ils sont incarnés, en phase d'élaboration et de mise en œuvre des OPATB, respectivement par l'Ademe et l'Anah. L'Anah remplit ses missions d'aides aux propriétaires bailleurs et aux occupants modestes, elle dispose d'une expérience étoffée des Opérations Programmées et notamment sur les choix et méthodes opérationnelles : ciblage des bâtiments, définition du périmètre, modalités d'accompagnement des propriétaires....

L'Ademe considère les OPATB (les opérations plus que le dispositif) comme des expérimentations faisant progresser les acteurs de la construction: «La multiplicité des cibles doit permettre de créer une véritable émulation des partenaires et favoriser les synergies [...] mobiliser tous les maîtres d'ouvrages et acteurs du bâtiment » (Ademe, 2009). L'Ademe anime le réseau d'échange d'expériences des OPATB, baptisé "Optinergie », et assure la gestion documentaire mise en ligne sur son site institutionnel.

Si l'OPATB a été calquée sur un dispositif spécifique à l'Anah, c'est selon une procédure chère à l'Ademe (l'appel à projet) que les collectivités sont choisies. Les critères sociaux habituels de l'Anah n'ont pas été pris en compte, seule comptait la capacité de la collectivité porteuse à mettre en œuvre l'OPATB. «Les cinq collectivités sorties lauréates de la présélection, par le jury, après examen de leur dossier de candidature, le 2 octobre 2002, ont démontré leur volonté de s'engager dans la procédure ambitieuse proposée par les OPATB. Elles ont réalisé en un temps réduit des études préalables couvrant un large champ d'informations, avec un grand niveau de précision dans la description des patrimoines concernés. $»^{13}$

Outre les différences de finalités qui leur sont assignées par l'État, ces deux agences nationales se distinguent par leurs modalités d'interventions. Distribuant des subventions depuis sa création en partenariat en partenariat avec des collectivités locales, l'Anah a des procédures bien cadrées et relativement lentes probablement dans un souci de traitement équitable des territoires. Accompagnant les acteurs innovants, l'Ademe 
privilégie la rapidité et l'efficacité ; elle s'octroie cependant la possibilité de suspendre un financement en phase opérationnelle.

\subsection{Enjeux et logiques d'action des collectivités locales partenaires de I'OPATB}

Remarquons la grande hétérogénéité des 16 OPATB en cours : périmètre $\left(2\right.$ à $\left.800 \mathrm{~km}^{2}\right)$, statut des porteurs (commune, intercommunalité, parc naturel régional, syndicat de Pays...), densité (rural à urbain), type d'habitat (agricole, maison individuelle, habitat collectif social ou privé...). La ville de Grenoble avec le soutien de la Métro répondit à l'appel à projet OPATB de février 2002 et fut sélectionnée avec quatre autres collectivités françaises. La ville d'Echirolles la rejoignit en 2004 lors du 3ème appel à projet. Fruit d'une dynamique régionale en matière de bâtiment durable (Roudil, 2008), 6 des 13 opérations mises en œuvre au niveau national en 2006 sont localisées en Rhône-Alpes dont 4 dans l'Isère.

Ville de Grenoble : un projet urbain devenu une illustration de la politique énergie/ climat communale

$\mathrm{Au}$ stade des études pré-opérationnelles, le périmètre couvrait une bande de 200 à 600 mètres autour de la nouvelle ligne de tramway traversant la ville. Trois cibles ont été retenues : bâtiments publics tertiaires, petits commerces et copropriétés d'habitat. Le périmètre de ces deux dernières a été restreint. Le volet «Petits commerces" concerne seulement les façades longeant la nouvelle ligne de tramway. Le périmètre du volet «Habitat » s'applique à l'intégralité des ilots de la ligne de tramway, c'est-à-dire aux bâtiments résidentiels situés sur les grands boulevards et dans les rues adjacentes à l'exception d'un tronçon.

Figure 1 : Périmètre et résultats OPATB Grands Boulevards

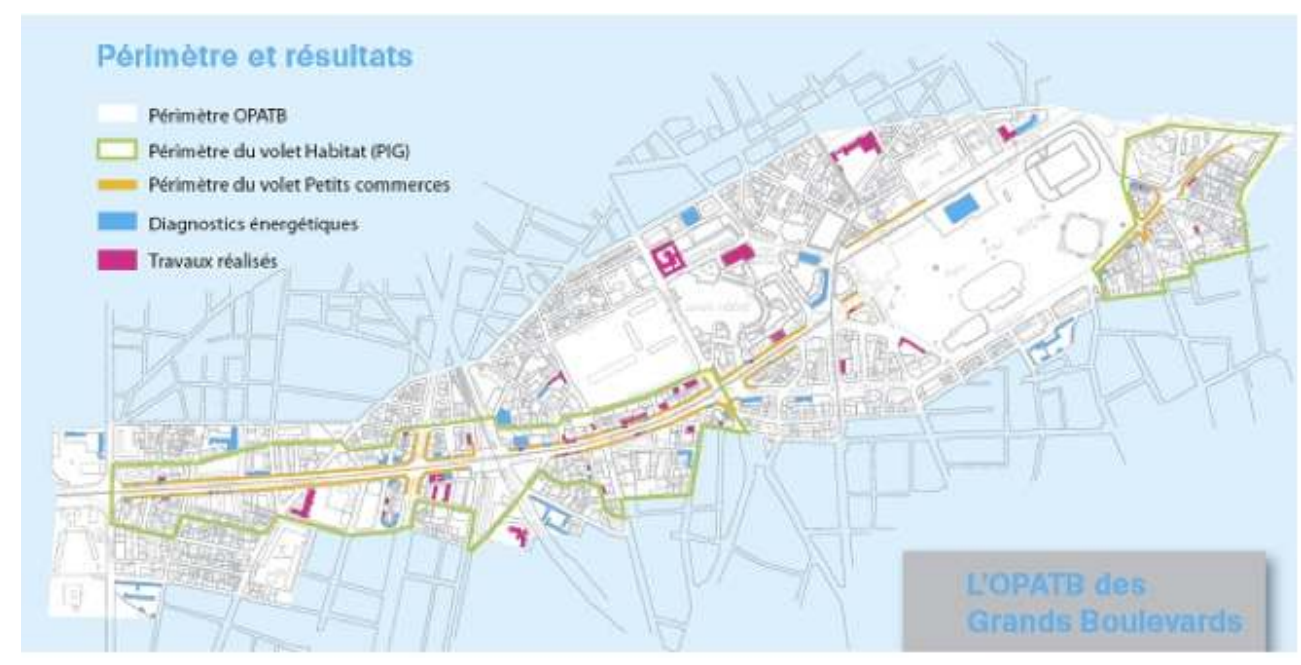

Source : Ville de Grenoble Bilan OPATB

Réalisés sur les anciennes fortifications qui ceinturaient le centre de Grenoble jusqu'en 1938 (Parent, 1982), les Grands Boulevards sont bordés par de hauts immeubles construits 
essentiellement entre 1945 et 1967. Leur performance énergétique est médiocre comme l'illustre la thermographie aérienne (Carré 2009).

Figure 2 : Thermographie aérienne secteur Grands Boulevards

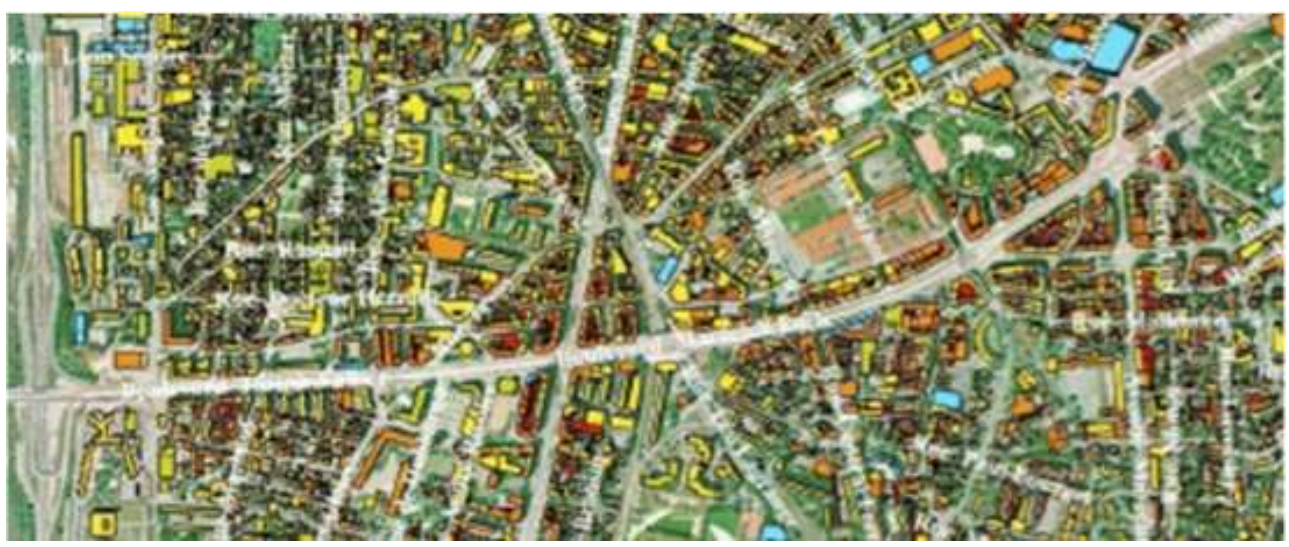

Les couleurs illustrent la température de surface de la toiture (bleu-jaune-orange-rouge du plus froid au plus chaud) révélant ainsi des niveaux de déperditions thermiques en période de chauffage.

Source : Carré, 2009 et Ville de Grenoble, 2007

Plusieurs grands projets ont concerné ce secteur de la ville : la démolition de l'estacade routière, la troisième ligne de tramway et la transformation apaisée du boulevard, la création d'un écoquartier à proximité (la caserne de Bonne) et le ravalement obligatoire des façades. L'ensemble de ces projets sont censés estomper la coupure urbaine entre le centre historique et les quartiers situés au sud composés d'anciens faubourgs pavillonnaires et industriels partiellement dénaturés par des grands immeubles construits au cours des trente glorieuses.

"L'intervention sur les Grands Boulevards s'inscrit dans un projet global de reconquête et de transformation d'un territoire» est-il souligné par le maire dans la plaquette de présentation de l'OPATB adressée aux habitants en 2006. Il s'agit d'unifier le territoire grenoblois et d'accompagner la réalisation de la ligne de tramway qui a, comme tout projet de cet ampleur, susciter les craintes voire l'opposition des commerçants et d'une fraction des riverains. Deux des trois volets ciblent ces catégories. Trois objectifs sont annoncés dans la plaquette: la redynamisation des commerces, l'amélioration thermique et énergétique des bâtiments, une architecture à valoriser. Le slogan de l'OPATB «nouvel air sur les Grands Boulevards " exalte autant les aspects esthétiques que climatiques.

En 2009, la ligne de tramway étant plébiscitée par les habitants du quartier et une majorité de commerçants bénéficiant des effets positifs, l'idéal de continuité urbaine véhiculée par l'OPATB a perdu de son utilité politique. Désormais, en dehors de la communication spécifique à l'OPATB, la ville présente, autant que faire se peut, l'OPATB comme une action phare du programme «Grenoble Facteur 4 » qui constitue de facto la version municipale du Plan Climat de l'agglomération et un nouvel idéal municipal ${ }^{14}$. Le renouvellement du conseil municipal de 2008 a entériné cette évolution en confiant son pilotage politique au maire-adjoint chargé du Développement Durable plutôt qu'à celui en charge de l'Urbanisme. 


\section{Ville d'Echirolles : une action dans un programme de développement durable complémentaire aux grands projets urbains}

Deuxième commune de l'agglomération, Echirolles s'est urbanisée à partir de 1945. Dans les années 1960 ; les immeubles d'habitat social et de copropriétés ont commencé à pousser à côté des pavillons ouvriers dans des secteurs séparés par des sites industriels. La ville s'est appuyée sur l'arrivée du tramway en 1987 pour créer en un quart de siècle un véritable centre urbain à l'épicentre du territoire communal.

Plus récemment et parallèlement, la municipalité a lancé au début des années 2000 un agenda 21 qu'elle utilise aussi pour mobiliser la population autour d'objectifs partagés en privilégiant les synergies entre le social et l'environnement. Elle mène, depuis lors, une démarche systématique de suivi des actions avec les habitants en veillant à couvrir à la fois la diversité des thématiques sectorielles, l'ensemble des quartiers de la ville et les différentes catégories d'habitants.

Figure 3 : Périmètre et affiche de l'OPATB Echirolles ouest

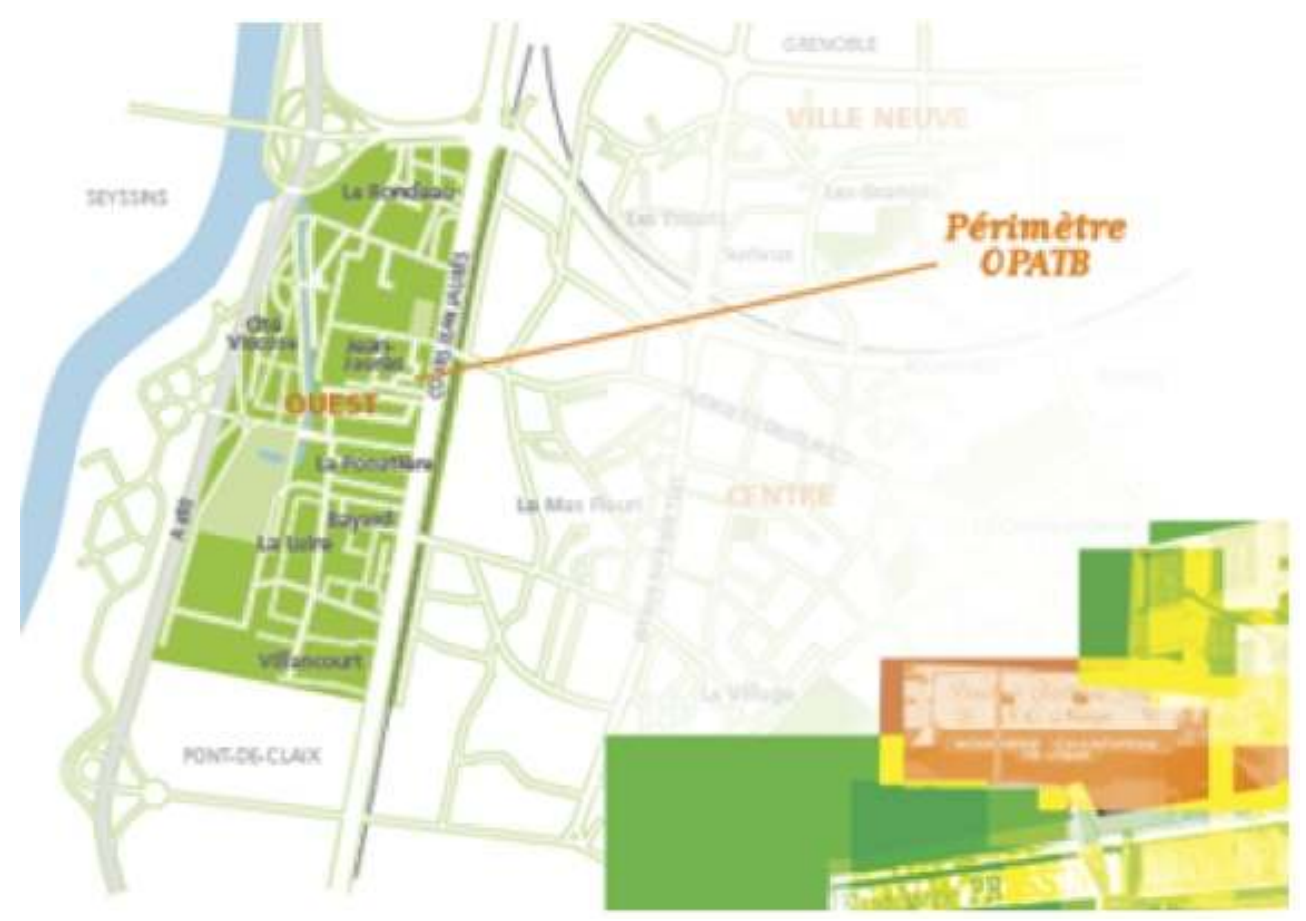




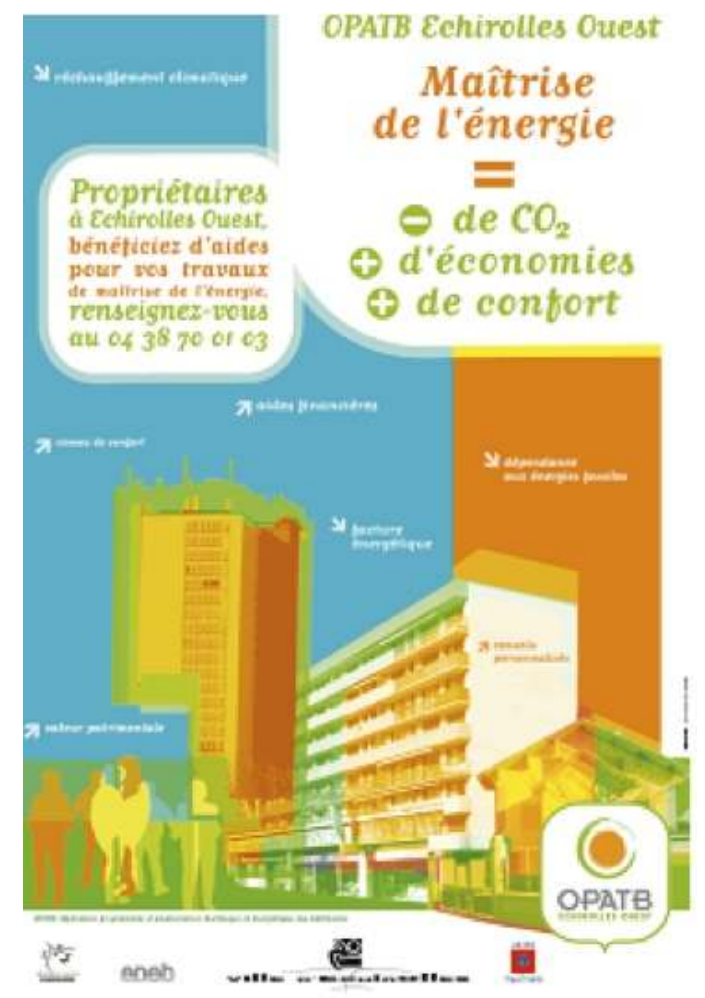

Source : ville d'Echirolles

L'OPATB «Echirolles Ouest» comble opportunément les creux de l'agenda 21 et plus généralement l'action politique de la ville :

isolés du reste de la commune par des infrastructures industrielles et de transport, les quartiers ouest ne bénéficient pas de la proximité des nombreux équipements publics du nouveau centre d'Echirolles;

les diverses actions « bâtiments » menées par la ville dans la lignée de l'agenda 21 ne concernaient pas les bâtiments existants privés.

Les quartiers ouest sont justement ceux où prédomine l'habitat privé (essentiellement collectif) et où les relais politiques du parti majoritaire sont moins développés. Par comparaison avec Grenoble, la communication sur l'OРATB insiste sur l'intérêt des particuliers et, implicitement, la solidarité planétaire, ignorant la dimension urbaine.

\section{La communauté d'agglomération Grenoble Alpes Métropole : embrasser une nouvelle compétence et expérimenter pour généraliser}

Partenaires des OPATB de Grenoble et Echirolles, Grenoble Alpes Métropole (appelée communément «La Métro») abonde sur son budget propre les subventions des communes, et, par délégation de l'État, les aides individuelles à l'amélioration de l'habitat privé selon des modalités cadrées par l'Anah. Sa faiblesse politique, intrinsèque aux modalités d'élection des conseillers communautaires, et le fait qu'elle ne dispose pas de la compétence urbanisme ${ }^{15}$ limitent cependant son influence sur la transformation de la ville.

Elle a créé en 1999 l'Agence Locale de l'Énergie, qui joue les rôles d'Espace Info Énergie et d'expertise/conseil, voire d'aiguillon, auprès des services de la Métro et des partenaires locaux. La Métro fut l'une des premières agglomérations françaises à élaborer un Plan 
Climat Local (présenté en mai 2005). Cette initiative a eu pour effet d'introduire l'objectif d'atténuation du changement climatique dans sa politique de l'habitat, et de mobiliser les communes, selon un mode davantage participatif que planifié (Debizet, 2009). Elle pilote le dispositif « Campagne isolation » qui succèdera aux OPATB à compter de 2010.

Restreinte à un rôle d'animation et d'incitation en matière d'urbanisme, la Métro entend initier et faciliter l'innovation ${ }^{16}$ au sein de la filière bâtiment qui demeure l'un des principaux secteurs économiques de l'agglomération dont les recettes sont assurées par la taxe professionnelle.

\section{D'autres acteurs contribuent au financement des OPATB}

La Région Rhône-Alpes cofinance les diagnostics énergétiques dans le cadre de dispositifs de «droit commun ». Sa part est minime dans le financement des travaux, elle concerne les panneaux solaires d'eau chaude et les panneaux photovoltaïques ${ }^{17}$. Outre la réduction des émissions de gaz à effet de serre, l'action de la Région s'inscrit dans une démarche de développement de la filière solaire et de l'écoconstruction (Roudil, 2008). Non-signataire des conventions OPATB, la Région n'a pas participé à leur ciblage ;

Le Conseil Général devait financer les chauffe-eau solaires des bâtiments publics et des copropriétés et la rénovation des bâtiments communaux. Cet apport s'est avéré très modeste dans le financement de l'OPATB ;

Dans le cadre du programme européen Concerto ${ }^{18}$, la Communauté Européenne finance un dixième de l'animation de l'OPATB Grands Boulevards ainsi que des travaux sur le parc social à Echirolles. Ce financement fut acquis quelques mois avant la signature de la convention, il a peut être permis des choix plus ambitieux de la part de la ville de Grenoble.

\subsection{Une mise en œuvre longue et complexe}

Il se sera écoulé une dizaine d'années entre la préparation du premier appel à projet national et l'aboutissement de la rénovation énergétique des premiers bâtiments grenoblois. Trois années ont été nécessaires pour mettre au point l'OPATB des Grands Boulevards (deux ans pour celle d'Echirolles Ouest). La convention de mise en œuvre signée par la ville de Grenoble, l'Ademe, l'État et Grenoble Alpes Métropole ${ }^{19}$ en octobre 2005 précise les objectifs quantitatifs, les engagements financiers, les règles de subvention ainsi que les modalités d'animation, de suivi, d'évaluation et de pilotage. Elle avait été précédée par des études préalables, le choix des prestataires pour l'animation ${ }^{20}$ et les études pré-opérationnelles ainsi qu'un «test» du dispositif sur des copropriétés. L'évolution parallèle des dispositifs fiscaux nationaux et des aides de droit commun des grandes collectivités a retardé la mise au point du système d'aide et la répartition des financements.

Une fois signée la convention, la phase opérationnelle commence par un long et fastidieux travail d'information des commerçants, des syndics et des conseils syndicaux pour les inciter à lancer un diagnostic énergétique. Le schéma ci-dessous illustre le processus de décision combiné de la ville et des copropriétés. Le syndic de copropriété et le conseil syndical sont accompagnés pendant deux ans jusqu'au vote des travaux en assemblée générale. Même lorsque les travaux sont communs à la copropriété, une part non négligeable des subventions est versée individuellement aux copropriétaires. 


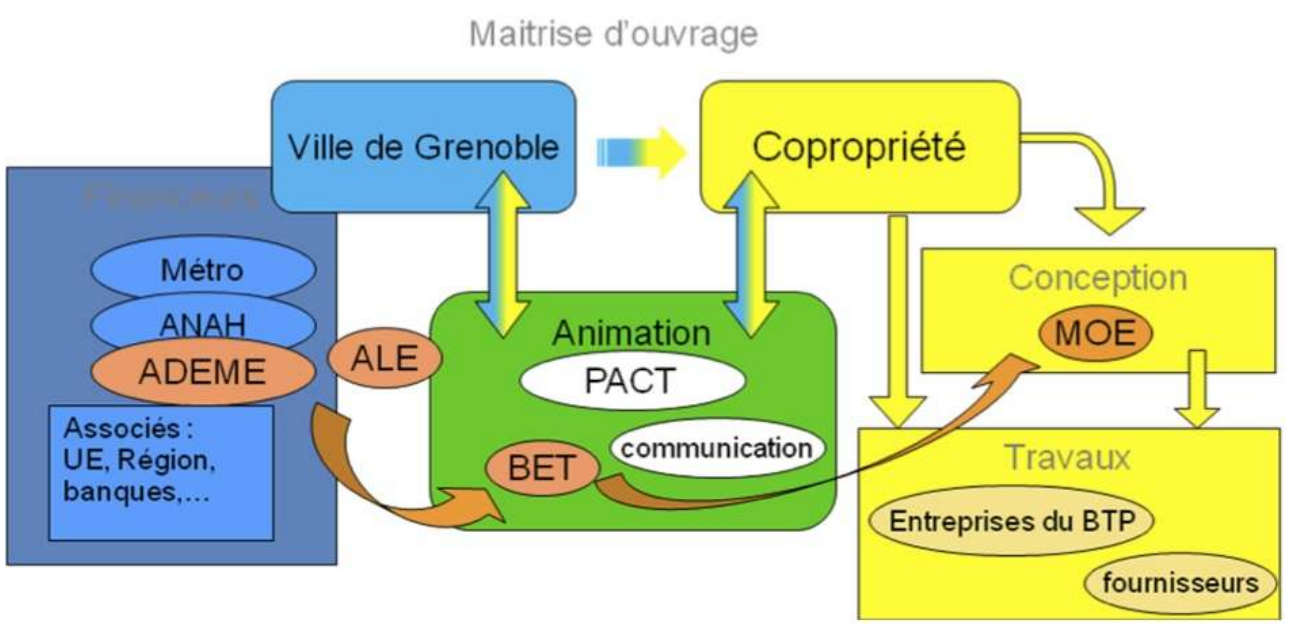

Source : G. Debizet à partir d'un schéma d'E. Carré, PACT de l'Isère, 2009

Financé par les signataires de la convention OPATB et ses partenaires (en bleu), le dispositif d'animation (en vert) a pour finalité de conduire les copropriétés (en jaune) vers la maîtrise d'ouvrage de travaux de rénovation: chaque copropriété recrute un maître d'œuvre puis une entreprise de travaux. Chaque catégorie dispose d'une expertise technique (en orange).

L'animation de l'OPATB combine l'apport de connaissances techniques, organisationnelles et financières aux copropriétés et aux copropriétaires et la gestion (communication et montage des demandes) des aides aux travaux. Le PACT de l'Isère, spécialisé dans l'accompagnement de l'amélioration de l'habitat, a assuré la coordination de l'ensemble des prestataires composant l'équipe d'animation (BE techniques, communication...).

\section{Les OPATB comme apprentissage collectif multiscalaire et interprofessionnel}

Cette description s'appuie sur l'OРАTВ Grands Boulevards dont un bilan a été réalisé fin 2009 par les services de la ville de Grenoble avec la contribution de l'Agence Locale de l'Énergie ${ }^{21}$. Voici en quelques chiffres les objectifs et les principaux résultats de l'OPATB des Grands Boulevards. Elle se focalise sur le volet «habitat» qui correspond intégralement à des copropriétés.

Tableau 1 : L'OPATB Grands Boulevards en chiffres

\begin{tabular}{|l|l|l|l|}
\hline Indicateur du volet Habitat (copropriétés) & Unité & $\begin{array}{l}\text { Programmé } \\
\text { Convention } \\
2005^{22}\end{array}$ & $\begin{array}{l}\text { Réalisé Bilan 2009 } \\
\text { estimation }\end{array}$ \\
\hline $\begin{array}{l}\text { Nombre de copropriétés contactées/diagnostiquées } \\
\text { (sur un total de 220 dans le périmètre) }\end{array}$ & - & $\mathbf{8 0 / 5 1}$ \\
\hline
\end{tabular}




\begin{tabular}{|c|c|c|c|}
\hline Nombre de copropriétés engageant des travaux & & 22 & 23 \\
\hline Nombre de copropriétés aidées par l'Ademe & & - & 12 \\
\hline Nombre de logements concernés & & 650 & 637 \\
\hline $\begin{array}{l}\text { Montant des travaux }{ }^{23} \text { HT y compris maîtrise } \\
\text { d'œuvre }\end{array}$ & $\mathrm{k} €$ & 3300 & 5230 \\
\hline Coût animation/formation/évaluation (estimation ${ }^{24}$ ) & $\mathrm{k} €$ & 555 & 620 \\
\hline Aides publiques mobilisées pour les travaux ${ }^{25}$ & $K €$ & 1143 & 1580 \\
\hline $\begin{array}{l}\text { Taux moyen de subvention aide travaux/montant } \\
\text { travaux }\end{array}$ & $€ / €$ & $35 \%$ & $30 \%$ \\
\hline $\begin{array}{l}\text { \% d'aide des propriétaires bailleurs par rapport aux } \\
\text { travaux (par rapport au surinvestissement isolation) }\end{array}$ & $\%$ & - & $\begin{array}{l}35 \text { à } 80 \\
(50 \text { à } 120)\end{array}$ \\
\hline $\begin{array}{l}\text { \% d'aide des propriétaires occupants par rapport aux } \\
\text { travaux (par rapport au surinvestissement isolation) }\end{array}$ & $\%$ & - & $\begin{array}{l}20 \text { à } 80 \\
(40 \text { à } 150)\end{array}$ \\
\hline $\begin{array}{l}\text { Économie d'énergie primaire prévisionnelle } \\
\text { (estimation en phase conception) }{ }^{26}\end{array}$ & Tep/an & 127 & 244 \\
\hline Efficacité énergétique des travaux & $\begin{array}{l}\mathrm{k} € / \\
\text { tep/an }\end{array}$ & 26 & 21,4 \\
\hline $\begin{array}{l}\text { Réduction des émissions de } \mathrm{CO}_{2} \text { (estimation en phase } \\
\text { conception) }\end{array}$ & $\begin{array}{l}\mathrm{tCO}_{2} / \\
\text { an }\end{array}$ & 112 & 575 \\
\hline Efficacité carbone des travaux & $\begin{array}{l}\mathrm{k} € / \mathrm{tCO} \\
\text { 2/an }\end{array}$ & 29,4 & 9,1 \\
\hline Efficacité carbone de l'aide publique aux travaux & $\begin{array}{l}\mathrm{k} € / \mathrm{tCO} \\
2 / \mathrm{an}\end{array}$ & 10,2 & 2,7 \\
\hline $\begin{array}{l}\text { Efficacité carbone de l'aide publique aux travaux }+ \\
\text { animation }\end{array}$ & $\begin{array}{l}\mathrm{k} € / \mathrm{tCO} \\
{ }_{2} / \mathrm{an}\end{array}$ & 15,2 & 3,8 \\
\hline
\end{tabular}

Sources : convention (Anah 2005) et bilan (Ville de Grenoble 2009)

Finalement, le montant des travaux votés par l'ensemble des copropriétés s'avère beaucoup plus élevé que prévu (5,8 contre 3,3 M€). L'écart envisagé/réalisé des économies d'énergie est du même ordre (244 contre 127 Tep/an), alors que le montant d'aides augmente mais dans des proportions beaucoup plus faibles (1,53 contre $1.1 \mathrm{M} €$ ). Globalement, les aides publiques OPATB se sont révélées plus efficaces que prévues : elles s'élèvent à 6,3 k€ (contre 8,7 prévu) par Tep/an économisé27.

L'écart est encore plus marquant rapporté aux émissions de $\mathrm{CO}_{2}$ attendues après travaux : 575 tonnes de $\mathrm{CO}_{2}$ économisées contre 112 envisagées. Chaque tonne de $\mathrm{CO}_{2}$ économisé annuellement a couté en moyenne aux partenaires financeurs de l'OPATB $2700 €$ (près de 
$4000 €$ si l'on tient compte aussi du coût de l'animation) au lieu de $9820 €$ de subvention publique prévue initialement. L'efficacité de l'argent public en termes de réduction de GES s'avère beaucoup plus élevée que prévue. Cela est dû au fait que les études préopérationnelles avaient surestimé la proportion des copropriétés chauffées par l'électricité.

\subsection{Une contagion tardive et plafonnée}

Les décisions des copropriétés sont rythmées par les assemblées générales annuelles. Le financement intégral du diagnostic par l'OPATB a permis de lancer cette étude sans solliciter l'accord de l'assemblée générale. Si le conseil syndical de la copropriété en prend l'initiative, l'assemblée générale suivante prend connaissance du diagnostic, et sélectionne un maître d'œuvre. Un an plus tard, une deuxième assemblée choisit le scénario de rénovation et l'entreprise qui réalisera les travaux (Carré, 2009). Entre temps, l'équipe d'animation de l'OPATB aura élaboré le plan de financement à l'échelle de la copropriété et informé tous les copropriétaires qui le souhaitent sur la part des aides dont ils peuvent bénéficier individuellement.

Figure 5 : Chronologie des décisions des copropriétés OPATB Grands Boulevards

\section{Evolution projets habitat}

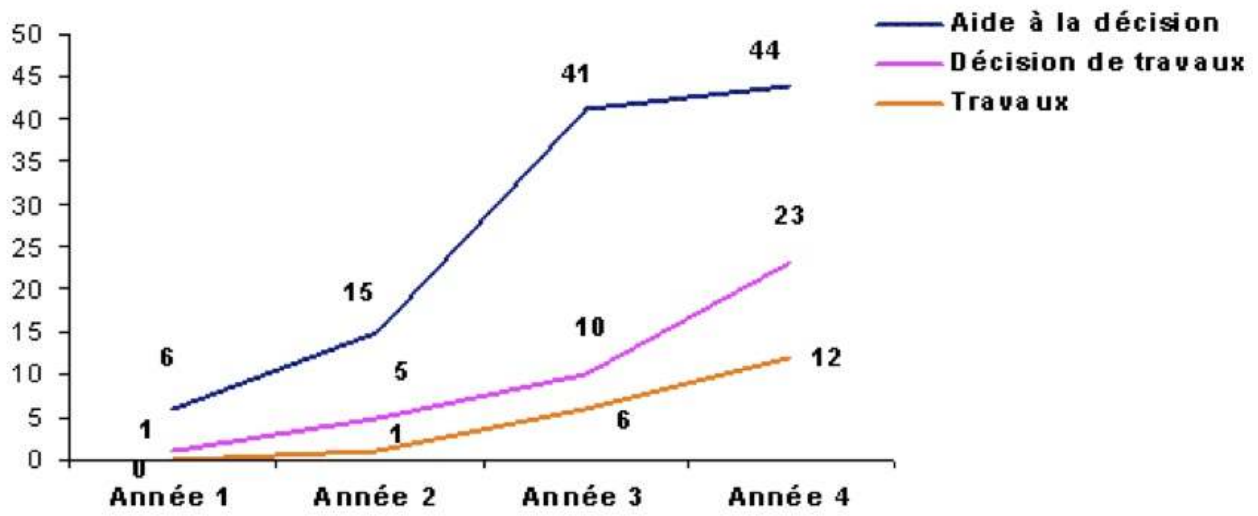

Source : Ville de Grenoble et comité de pilotage du 27/11/2009

La forme exponentielle de la courbe «Décision de travaux » caractérise la diffusion des innovations par contagion. L'échéance de la fin de l'OPATB (seuls sont financés les travaux décidés avant la fin de la $4^{\mathrm{e}}$ année) a conduit le comité de pilotage à cesser de financer des diagnostics au-delà de la $3^{\mathrm{e}}$ année, ce qui explique le tassement de la courbe "Aide à la décision ». Contrairement à la convention qui prévoyait un lissage des travaux sur 4 ans, plus de la moitié des travaux démarrent la $4^{\mathrm{e}}$ année et un quart l'année suivante. Le fait de fixer une limite temporelle à l'octroi des subventions a pour conséquence de concentrer les décisions la dernière année par un double effet de report des décisions qui auraient pu être techniquement prises auparavant et d'accélération des décisions avant la clôture du subventionnement. 


\subsection{Une convergence forte et non-prévue vers l'isolation par l'extérieur}

La convention de 2005 régissant les relations entre tous les partenaires publics financeurs prévoyait $3300000 €$ de travaux ainsi répartis :

Isolation extérieure pour 4 copropriétés (480 logements) ;

Remplacements des menuiseries extérieures pour 6 copropriétés (260 logements) ;

Eau chaude sanitaire solaire pour 2 copropriétés (60 logements) ;

Travaux divers en partie commune, (régulation collective du chauffage, isolation des conduits, ventilation...) pour 10 copropriétés (400 logements) ;

Travaux d'amélioration énergétique en partie privative (isolation par l'intérieur, régulation individuelle...) pour 120 logements.

Figure 6 : Déperditions thermiques d'un immeuble non isolé

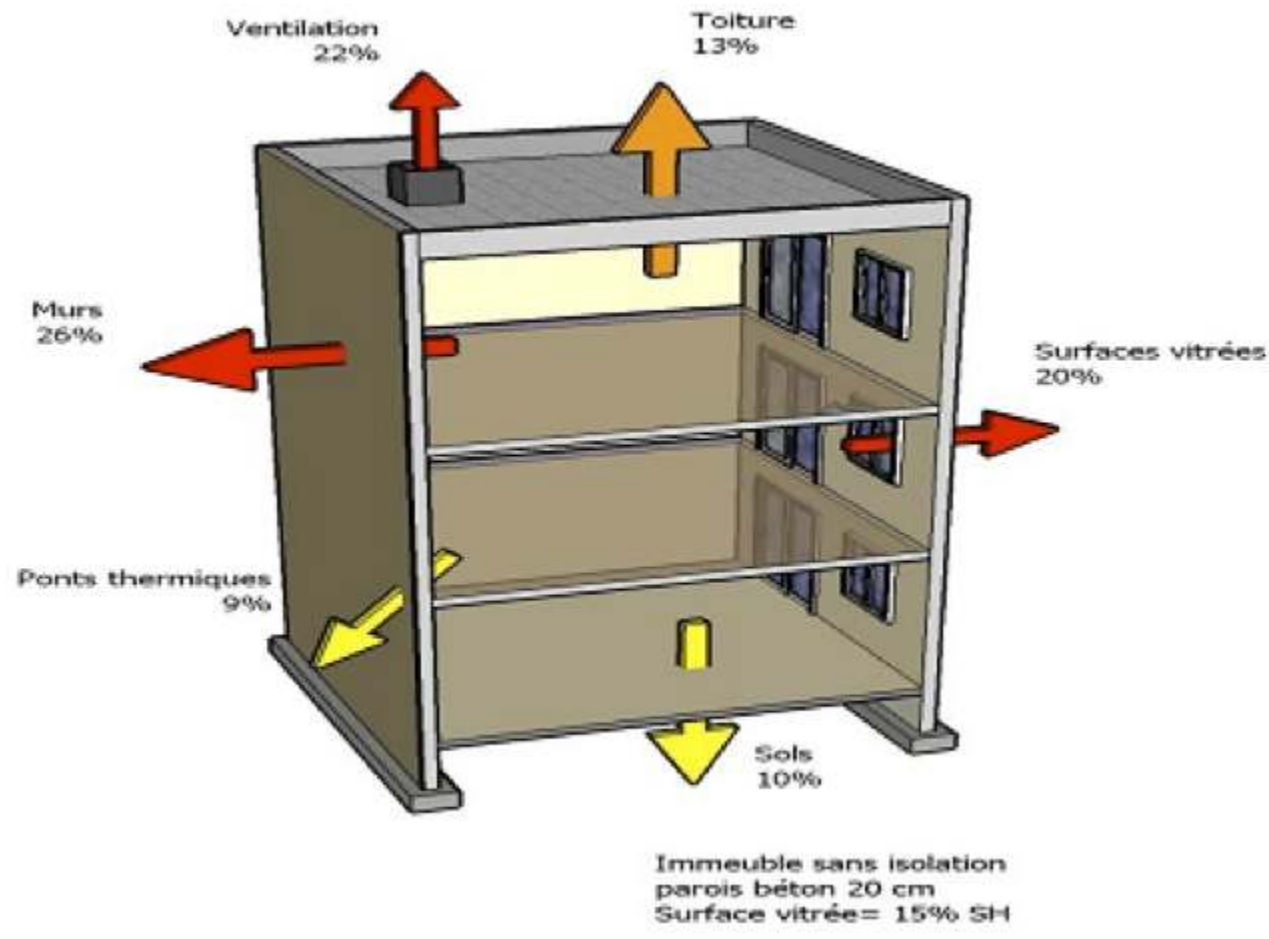

Source : Ademe

Tous les postes de déperditions thermiques d'un immeuble (cf. figure ci-dessus) avaient été programmés. Au moment du bilan de l'OPATB, il s'avère que la totalité des 23 copropriétés se sont engagées dans l'isolation des façades et, pour moitié d'entre elles, des toitures ou planchers en plus. Seules deux ont, en outre, engagé des travaux sur le système de chauffage ou les menuiseries. Le renoncement à d'autres travaux que l'isolation thermique s'est opéré dès la deuxième année : la ville de Grenoble, l'Agence Locale de l'Énergie et l'équipe d'animation ont pris conscience des difficultés d'interventions coordonnées dans les parties privatives. "La première évolution a été une modification de l'éventail de solutions proposées. Avec la réalisation des diagnostics énergétiques, certains types de travaux sont apparus très complexes et chers à mettre en œuvre compte tenu des 
caractéristiques des bâtiments. En particulier, les travaux sur le système de ventilation ont été abandonnés, ainsi que les projets de commandes d'huisseries ${ }^{28}$ pour les parties privatives " (Carré, 2009). Ces travaux ont cessé rapidement d'être proposés parmi les scénarios présentés en assemblée générale. L'isolation par l'extérieur s'est avérée très attractive lorsque l'immeuble est soumis à l'obligation de ravalement. Les travaux nécessaires au ravalement (échafaudage, préparation des murs et enduit) représentent entre la moitié et les deux tiers de l'ensemble des travaux d'isolation thermique par l'extérieur (Source: Ville de Grenoble, présentation au comité de pilotage du 27 novembre 2009.). Autrement dit, entre la moitié et les deux tiers du coût des travaux d'isolation par l'extérieur doivent être de toute façon être dépensés en cas de ravalement. Finalement, par le jeu des subventions de l'OPATB, de nombreux copropriétaires ont dépensé moins d'argent pour l'isolation extérieure du bâtiment (tous postes confondus) que s'ils avaient décidé (et financé) le seul ravalement ${ }^{29}$. En toute logique financière, les copropriétés contraintes au ravalement sont surreprésentées (19 sur 23) parmi celles ayant engagé des travaux OPATB.

\subsection{Une animation et une gestion administrative lourdes}

Sur l'ensemble de l'OPATB Grands Boulevards (habitat + tertiaire + commerce), le coût de l'animation s'élève comme prévu à 1 million d'euros. Le faible intérêt porté par les commerçants à la rénovation énergétique de leur local et le renoncement rapide de plusieurs maitres d'ouvrage des bâtiments tertiaires ont conduit la ville à redéployer le budget animation commerce et tertiaire vers l'habitat.

Le travail d'animation fut plus complexe qu'envisagé. Pour chaque copropriété, il a fallu mettre au point une plaquette décrivant de façon simplifiée les travaux et les financements: un indispensable travail de "traduction» des propositions du maitre d'œuvre et du dispositif administratif de l'OPATB. Conditionnées à des performances de consommation énergétique qui ne pouvaient être garanties au moment du vote des travaux par l'assemblée générale, les aides de l'Ademe ont peu été prises en compte par les copropriétaires ${ }^{30}$. L'adossement au ravalement obligatoire (aidé à hauteur de $20 \%$ par la ville de Grenoble) n'a pas donné lieu à des synergies dans le traitement des dossiers. Le délai entre la réception du programme de travaux envisagés par le conseil syndical et l'assemblée générale s'est souvent avéré trop court pour que l'animateur de l'OPATB fournisse un plan de financement aux copropriétaires qui le souhaitaient; ces derniers n'ont pu solliciter un prêt bancaire dans des délais aussi brefs.

Ces difficultés pourraient expliquer l'absence de majorité pour les travaux d'isolation au sein de plusieurs copropriétés. Inversement, l'on peut se demander si le dépassement de ces difficultés dans un futur dispositif ne permettrait pas d'atteindre les majorités requises avec un montant d'aide publique à la copropriété sensiblement moindre. Précisons que l'enveloppe des aides publiques à la copropriété fut nettement plus élevée que celle des aides individuelles aux copropriétaires.

\subsection{Confrontation des cultures professionnelles}

Les différences de logiques observées au niveau national (efficacité environnementale pour l'Ademe et efficacité socio-économique pour l'Anah) s'estompent localement en phase opérationnelle. L'implication des élus de la ville dans le comité de pilotage et la 
démocratie locale contribuent à centrer l'attention des professionnels sur les habitants. Le caractère pionnier du dispositif, la relative jeunesse des techniciens les plus directement impliqués et le respect que se portent mutuellement leurs chefs de service plus expérimentés ont facilité la fabrication d'un bien commun, au sens de Muller (Muller, 2000) articulant «idéalement» les dimensions environnementale, sociale et économique.

La répartition des activités présentent des similitudes avec celles décrites entre l'Anah et l'Ademe. Le service « réhabilitation » de la ville de Grenoble a porté l'OPATB ${ }^{31}$. L'Agence Locale de l'Énergie de l'agglomération grenobloise (ALE) a apporté son expertise et contribué à légitimer les experts techniques agissant auprès ou pour le compte des copropriétés (cf. figure 4). Contrairement à l'Ademe, l'ALE n'attribue pas de subventions ; elle a cependant conseillé la Métro dont l'apport financier fut loin d'être négligeable.

Le laborieux ajustement du dispositif d'accompagnement tint essentiellement à la différence entre le modèle de décision des collectivités et celui des copropriétés. La démarche de programmation des collectivités s'appuie sur des expertises exhaustives permettant de préparer minutieusement les décisions budgétaires et les actes écrits. Côté copropriété, la " gestion patrimoniale » consiste à étaler dans le temps les travaux de gros entretien et, de facto, à reporter bien souvent les investissements d'amélioration ; la prise de décision relève d'opportunités financières, symboliques (écoresponsabilité, standing) et démocratique (égard aux situations des membres de la " communauté », coalitions...).

Les maitres d'œuvre et les entreprises de réalisation ont aussi évolué. Le peu d'enthousiasme initial vis-à-vis de l'isolation extérieure de certains architectes chargés des ravalements a fait capoter des décisions de travaux les premières années (Carré, 2009). Le nombre d'entreprises en mesure d'assurer des travaux d'isolation par l'extérieur s'est accru entre 2006 et $2009^{32}$ à tel point que le prix du $\mathrm{m}^{2}$ d'isolant extérieur posé aurait chuté de $25 \%$ en $2009^{33}$.

\section{Généralisation prochaine de la rénovation énergétique à l'agglomération}

Délégataire des aides de l'Anah et pilote du Plan Climat local, Grenoble Alpes Métropole a préparé de longue date un élargissement de l'aide aux copropriétés à l'ensemble de l'agglomération. Dès 2006, elle a mis au point une exposition itinérante dans les communes de l'agglomération intitulée "Campagne isolation». En 2007, des études menées par l'ALE, l'Agence d'Urbanisme de la Région Grenobloise et l'université (Daniel et al., 2007) convergent pour un traitement prioritaire des bâtiments d'habitat collectif des années 45 à 75 . Ces bâtiments très déperditifs représentent près de la moitié des logements de l'agglomération Dans l'ensemble, ils se caractérisent par des façades simples sans modénature, et des toitures-terrasses planes. On ne leur attribue pas une grande qualité architecturale. Ils se situent à Grenoble et dans les communes de la première couronne, socialement moins favorisées que celles de la deuxième couronne. 
Figure 7 : Logements de l'agglomération grenobloise par année de construction et par commune

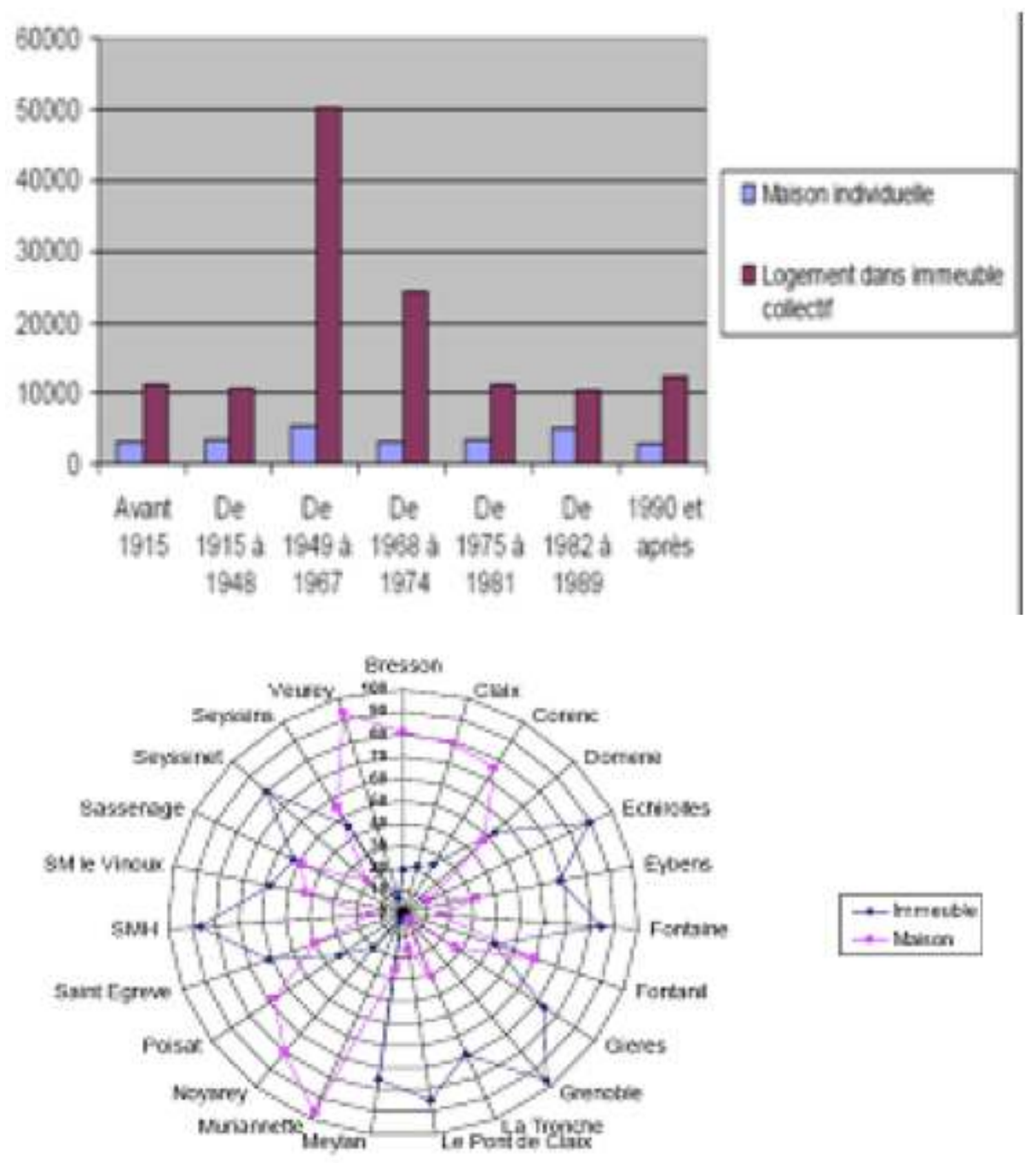

SOURCE : DANIEL ET AL, 2007, À PARTIR DE DONNÉES AURG

En février 2008, une délibération du conseil communautaire définit les orientations. Les modalités d'élaboration d'un nouveau dispositif sont adoptées en mars 2009 par le conseil communautaire. Des expertises techniques et une concertation des collectivités, de l'Ademe et des fournisseurs d'énergie ont permis de définir les principes et les rôles respectifs des partenaires.

\subsection{Un dispositif qui tient compte des enseignements de l'OPATB}

Entre 2010 et 2013, la Métro vise la réhabilitation énergétique de 150 copropriétés (5 000 logements sur un total de 60000 éligibles) construites entre 1945 et 1975. Préparée par le vice-président chargé de l'environnement et du développement durable, la délibération dénommée Campagne isolation ${ }^{34}$ énonce quatre principes :

Simplifier les procédures en instaurant un guichet financier unique ; Impulser une dynamique auprès des professionnels par des formations techniques ; Ne pas tuer le gisement, en imposant une obligation de moyens pour chaque poste de travaux; 
Se faire la main sur le parc de bâtiment à faible richesse architecturale.

Concrètement, des bouquets de travaux sont prédéfinis : ils concernent l'isolation des façades, des toitures et des planchers, le changement de menuiseries ainsi que des actions d'accompagnement sur le poste ventilation. Le taux de subvention le plus bas s'applique à la simple isolation des façades, il s'accorde bien au ravalement. Le taux le plus fort, dit Rénovation exemplaire est calé sur un appel à projet régional financé par la Région, l'Ademe et l'Anah «Bâtiments démonstrateurs à basse consommation énergétique » qui correspond à la performance " $\mathrm{BBC}$ rénovation énergétique». Chaque commune est invitée à participer financièrement au dispositif Campagne isolation à hauteur de la moitié de l'engagement de la Métro. Dans le cas contraire, le taux de subvention attribué aux copropriétés sera réduit de facto d'environ un tiers.

\subsection{Un compromis évolutif entre l'efficacité carbone et l'équité sociale}

Les aides individuelles aux propriétaires étant définies par un cadre national et réservées aux occupants modestes et aux bailleurs, la marge de manœuvre des collectivités locales, et donc de la Métro, s'exerce sur l'aide à la copropriété. La meilleure efficacité carbone de l'enveloppe budgétaire consacrée localement à cette aide consisterait à aider les copropriétés dont les copropriétaires disposent globalement d'une capacité financière élevée et ne restreignent pas leur consommation énergétique. Le taux de subvention de cette aide serait alors calé pour faire basculer le copropriétaire supplémentaire permettant d'atteindre la majorité requise pour les travaux.

Cela ne serait pas sans poser des problèmes d'équité, car les propriétaires modestes de ces copropriétaires risquent de ne pouvoir assurer financièrement leur quote-part des travaux et seraient poussés à revendre leur logement. D'autre part, diriger l'argent public vers les copropriétés les plus favorisées de l'agglomération risquerait fort d'accroître les disparités économiques et sociales entre les quartiers et entre les communes.

Une clause de la campagne isolation répond à ces risques, elle fait remonter les arbitrages jusqu'au conseil de communauté : "... le Conseil de Communauté sera consulté sur une programmation pluriannuelle, mise à jour chaque année et veillera à ce que les volumes de copropriétés subventionnées soient répartis de manière équilibrée entre les différents territoires de l'agglomération. $»^{35}$. Il n'est pas précisé si une démarche proactive sera engagée vers des copropriétés moins favorisées. Cette décision relèvera du comité de pilotage et de comités techniques auxquels seront associés des représentants des communes concernées. C'est donc finalement au fur et à mesure que seront élaborés les compromis entre efficacité énergétique carbone et équité sociale.

\section{Conclusion}

Sur un périmètre constitué de bâtiments d'habitation réalisés en plein boom de l'urbanisation précédent la réglementation thermique des bâtiments, l'isolation thermique par l'extérieur s'est avérée opportune compte tenu de la gouvernance des copropriétés et de l'obligation de ravalement. En moyenne, $10000 €$ ont été dépensés ${ }^{36}$ pour réduire d'une tonne les émissions annuelles de $\mathrm{CO}_{2}$. Il est vraisemblable que le coût de l'isolation par l'extérieur baissera parallèlement au développement de l'offre de 
travaux qualifiés. Cependant, il ne faut espérer des coûts sensiblement plus bas, d'une part car la fabrication des matériaux isolants est déjà industrialisée et d'autre part parce que la diversité des bâtiments à rénover, si ce n'est la demande de qualité architecturale et urbaine, exclut une industrialisation de la pose d'isolant.

Il est vraisemblable que les aides publiques restent le levier prépondérant de la rénovation énergétique de l'habitat collectif privé pendant plusieurs années. L'incitation publique territorialisée est indispensable tant sous forme d'apport de connaissances et d'animation que d'aides financières aux travaux. Sans compter les dispositions fiscales, environ $4000 €$ ont été investis ${ }^{37}$ par les partenaires publics de l'OPATB de Grenoble pour réduire d'une tonne les émissions annuelles de $\mathrm{CO}_{2}$. Le nouveau dispositif campagne isolation de l'agglomération grenobloise vise à réduire le coût de l'animation (guichet unique et simplification des diagnostics des bâtiments) et celui des travaux par une action à l'échelle du bassin urbain.

Le débat restera ouvert sur le financement public et les modalités d'attribution des aides. Comme au niveau national, les collectivités sont confrontées à la contradiction entre les objectifs environnementaux et sociaux. Maximiser la réduction des émissions de GES d'une enveloppe budgétaire conduit à flécher les crédits publics vers les copropriétés les plus aisées. Le même risque peut être identifié à propos de l'allocation des aides nationales aux différents territoires par la procédure d'appel à projet.

Les opérations programmées relèvent d'une gouvernance interterritoriale et intersectorielle complexe et illustrent la subtilité des ajustements. Par comparaison, l'on peut s'interroger sur l'uniformité des modalités fiscales sur le territoire national et l'absence d'intermédiaire public entre le particulier et l'artisan. Entre accompagnement territorialisé et mécanismes économiques assis sur la fiscalité, les articulations sont à ajuster pour généraliser une rénovation énergétique socialement équitable et économe des deniers publics. Un chantier technique et politique pour plusieurs années à éclairer par les retours d'expériences d'autres opérations programmées.

\section{BIBLIOGRAPHIE}

Ademe, 2009, « Réhabilitation du parc locatif privé », Ademe \& vous, Stratégie \& études, $\mathrm{n}^{\circ} 18$, février

Aibar E., Bijker W.E., 1997, "Constructing a city: The Cerdà Plan for the Extension of Barcelona”, Science Technology Human Values, vol. 22, n 1, p. 3-30

Anah, 2009, «Les avantages fiscaux en faveur des logements du parc privé en 2009 », juin

Berdoulay V., Soubeyran O., 2002, L'écologie urbaine et l'urbanisme. Aux fondements des enjeux actuels, La Découverte, collection « Recherches », Paris

Besson L, 2000, Discours, Anah - Journée de l'amélioration de l'habitat, 5 décembre, mis en ligne le 15 décembre sur le site du ministère de l'Équipement, actuellement http:// www.logement.equipement.gouv.fr/article.php3 ?id_article =944, consulté le 20 octobre 2009 
Carré E., 2009, L'OРATB des Grands Boulevards Intervention sur les copropriétés d'avant 1975, mémoire PFE de master Urbanisme et Projet Urbains, sous la direction de Debizet G., Pact de l'Isère et Institut d'Urbanisme de Grenoble UPMF, septembre

CGDD (Commissariat Général au Développement Durable), 2009, Chiffres clés de l'énergie - Repères, décembre

Daniel J., Meglia D, Ren X., Porral G., 2007, Préparation d'une campagne isolation, Mémoire de master Maîtrise d'ouvrage et management de patrimoine bâti, sous la direction de Debizet G., Université de Grenoble, et de Derive G., Agence Locale de l'Énergie, avril

Debizet G., 2007, « Contribution d'un forum hybride local à l'expertise urbaine française », 43 Colloque de l'ASRDLF, Les dynamiques territoriales, débats et enjeux entre les différentes approches disciplinaires, Grenoble \& Chambéry, 11-13 juillet, http://halshs.archives-ouvertes.fr/ halshs-00265062/fr/

Debizet G., 2009, « Comment mobiliser les communes dans les Plans Climat Territoriaux ? ", Quatrièmes Rencontres Régionales « Énergie Climat » des collectivités de Rhône Alpes, Villeurbanne, 2 juin, référence halshs-00406521 sur le site http://halshs.archives-ouvertes.fr Gravejat A., 1991, «Sur les opérations programmées d'amélioration de l'habitat à Lyon : 1975-1985 », Revue de géographie de Lyon, vol. 66, n² 2, p. 115-121

Henry E., 2008, «L'expérience de Grenoble depuis 2006 », Introduction à l'atelier 3, Colloque Villes et constructeurs : vers un copilotage de la qualité environnementale des bâtiments, Grenoble juin, http://www.pacte.cnrs.fr/spip.php ?article899

Marchal J., 2008, Modélisation des performances énergétiques du parc de logements, synthèse du rapport de stage d'ingénieur des Ponts et Chaussées à l'Anah, sous la direction de Lagrande E.

Muller P., 2000, «L'analyse cognitive des politiques publiques : vers une sociologie politique de l'action publique », Revue française de science politique, vol. 50, n², p. 189-208

Pelletier P., Jacq A., Orphelin M. (sous la dir.), 2008, Rapport d'étape au ministre d'État, ministre de l'Écologie, du Développement et de l'Aménagement durables, Comité opérationnel « rénovation des bâtiments existants ", janvier

Parent J.F., 1982, Grenoble deux siècles d'urbanisation : projets d'urbanisme et réalisations architecturales 1815-1965, PUG, Grenoble

Roudil N., Marchand D., Laborgne P., Huber A., 2008, PEREBAT les politiques énergétiques développées par les régions dans le cadre du bâti, Rapport final CSTB, novembre

SESAC, 2006, Newsletter, $n^{\circ}$ 1, May, http://www.concerto-sesac.eu/spip.php? page $=$ newsletter\&id_rubrique $=2$

Star S. L., Griesemer J. R., 1989, “Institutional Ecology, 'Translations' and Boundary Objects: Amateurs and Professional in Berkely's Museum of Vertebrate Zoology, 1907-39”, Social Studies of Sciences, vol. $19, \mathrm{n}^{\circ} 3$, p. 387-420

Trompette P., Vinck D., 2009, « Retour sur la notion d'objet frontière », Revue d'anthropologie des connaissances, vol. $3, \mathrm{n}^{\circ} 1$, p. 5-27

\section{NOTES}

1. Selon l'Anah, $56 \%$ des occupants des résidences principales sont propriétaires et $21 \%$ locataires de propriétaires privés (Marchal, Lagrande, 2008). 
2. Comme le firent Aibar et Bijker à propos de la trame urbaine de Barcelone (Aibar, 1997).

3. Transcrivant en droit français une directive européenne, la loi sur l'énergie du 13 juillet 2005 a institué une première étape de réglementation dans l'existant. Le décret sur la performance énergétique des bâtiments existants 21 mars 2007 fixe des obligations de performance énergétique pour les bâtiments de plus de $1000 \mathrm{~m}^{2}$ faisant l'objet de travaux de réhabilitation importants (plus de $25 \%$ de la valeur du bâtiment hors foncier). Des décrets d'application concernent aussi des opérations de moindre importance, des exigences de performances minimales sont imposées lors du remplacement de composants de l'enveloppe (fenêtres, isolation thermique) ou d'équipements énergétiques, depuis le 1er novembre 2007.

4. Déduction fiscale pour travaux d'efficacité énergétique puis crédit d'impôt « développement durable » à partir de 2005, majoration du prêt à taux zéro en 2009 (Anah, 2009).

5. Citons principalement : renouvellement urbain, revitalisation rurale, copropriété et plan de sauvegarde, et programme social thématique (1990), sortie de vacances (1998), "Borloo ancien » (2006). Source : Anah, Les dispositifs programmés, page http://www.anah.fr/laction-territoriale/ les-dispositifs-programmes/ du site de l'Anah consultée le 20 octobre 2009.

6. Page http://www.anah.fr/lanah/les-chiffres-cles/ du site internet de l'Anah consulté le 20 octobre 2009.

7. Les $526 \mathrm{M} €$ de subventions distribuées en 2008 par l'Anah auraient engendré 2 milliards de travaux soit l'emploi de 35.000 personnes.

8. Dossier de Presse «Lancement des OPATB» du 20 février 2002, Ademe, MATE, Anah, secrétariat d'État au logement.

9. Précédée par de longues sections consacrées aux aspects sociaux et sanitaires et au renouvellement urbain, la référence aux "économies d'énergie » est intégrée dans la section «qualité technique dans l'habitat existant » avant un développement plus long sur la «sonorité tranquillité ».

10. C'est en cela que l'OPATB se distingue des OPAH dites "thermique » ou "maîtrise énergétique » ou « développement durable ».

11. Dossier de Presse «Lancement des OPATB» du 20 février 2002, Ademe, MATE, Anah, Secrétariat d'État au logement.

12. Outre une vigilance en termes de communication institutionnelle, les ministères évaluent la mise en œuvre des OPATB. Les CETE ont été chargés d'une enquête et d'une synthèse sur l'avancement des OPATB en 2008 et 2009.

13. Dossier de presse: «OPATB Les collectivités s'engagent au travers des OPATB dans le développement durable », 29 novembre 2002.

14. Grenoble facteur 4 est un programme d'actions visant la réduction des émissions de GES. Une bonne partie de ces actions étaient engagées depuis plusieurs années à l'image du nouveau PLU ou du guide de la Qualité Environnementale, Architecturale et Urbaine (Henry, 2008).

15. Comme la plupart des communautés d'agglomération et contrairement aux communautés urbaines, Grenoble Alpes Métropole n'a pas la compétence urbanisme : chaque commune définit les règles d'urbanisme et délivre les autorisations de construire.

16. L'agglomération grenobloise est souvent comparée à un laboratoire urbain, un espace où prennent corps les innovations en matière de politique et de technologie urbaines. C'est à Grenoble que fut inventé sous la pression des citoyens le premier tramway à plancher bas, invention qui contribua sans doute au redéploiement du tramway dans les villes européennes et mondiales (Debizet 2007). La liste serait longue pour désigner les "premières grenobloises » : rue piétonne au milieu des années 1960, piste cyclable, réhabilitation des quartiers anciens en maintenant les populations au cours des années 1970... jusqu'au quartier de la caserne de Bonne, premier Grand Prix national Écoquartier en 2009. 
17. La part des aides de la Région sur l'ensemble des travaux de rénovation de l'OPATB Grands Boulevards était estimée à $1 \%$ du montant total et $3 \%$ des aides publiques. Source : bilan OPATB, Ville de Grenoble.

18. Sous la conduite de la ville de Grenoble, des opérateurs publics de l'aménagement, de l'habitat et de l'énergie et Grenoble Alpes Métropole se sont associés avec Växjö en Suède et Delft aux Pays-Bas pour partager et diffuser les bonnes pratiques d'efficacité énergétique en milieu urbain. Grenoble s'est focalisée sur les énergies renouvelables (thermique ECS, photovoltaïque et hydraulique), l'efficacité énergétique des bâtiments ainsi que l'innovation et la mobilisation des parties prenantes (SESAC, 2006).

19. En son nom et au nom de l'Anah dont la Métro est délégataire.

20. Pour les Grands Boulevards, il est coordonné par le PACT de l'Isère (et par Habitat Territoires Conseil pour Echirolles ouest).

21. ОРАTB Grands Boulevards: les résultats Grenoble 2006-2009, Ville de Grenoble, novembre 2009 et ОРАTB Grands Boulevards bilan année 4 et bilan complet, Comite de pilotage du 27 novembre 2009 Ville de Grenoble et partenaires, 54 diapositives.

22. Convention de mise en œuvre OPATB et PIG Grands Boulevards 2005-2009, Ville de Grenoble, Ademe, État, Grenoble Alpes Métropole, 17 octobre 2005.

23. Ce montant comprend essentiellement les travaux d'isolation extérieure, coût de ravalement compris.

24. Estimation par l'auteur, après avoir réparti les dépenses d'animation communes aux trois thématiques au prorata des dépenses spécifiques.

25. Les aides représentent $26 \%$ du coût total des travaux et plus de $50 \%$ du surinvestissement isolation. Les aides mentionnées ici sont les subventions aux copropriétaires, elles ne comprennent pas les déductions fiscales ou crédits d'impôts auxquelles chaque copropriétaire peut prétendre.

26. L'économie d'énergie a été déterminée en pondérant l'écart de déperdition thermique des surfaces extérieures en période de chauffage, avant et après isolation, par le coefficient de conversion primaire/finale de l'énergie utilisée dans chaque bâtiment. La réduction des émissions de gaz à effet de serre a été estimée en pondérant cet écart par les coefficients Ademe de conversion $\mathrm{CO}_{2} /$ énergie finale. Dans la convention, il avait été estimé qu'il y aurait autant de bâtiments rénovés chauffés au gaz qu'à l'électricité.

27. La dépense publique annoncée ici se limite à l'aide aux travaux dans le cadre de l'OPATB, elle ne tient pas compte du coût de l'animation et des aides fiscales. L'abandon des travaux autres que l'isolation thermique pourrait expliquer la meilleure efficacité énergétique des aides publiques.

28. Contrairement aux parois porteuses et à la toiture, les menuiseries relèvent des parties privatives. Leur remplacement ne peut être imposé à un copropriétaire par le syndicat de copropriété. Les animateurs de l'OРATB ont proposé, sans succès, des achats groupés de menuiseries pour réduire leur coût. Une majorité de copropriétaires avait déjà remplacé les fenêtres simple vitrage d'origine. Les autres préféraient différer ses travaux, leur capacité financière étant saturée par les dépenses d'isolation adoptées par la copropriété.

29. La ville de Grenoble a finalement ajusté les aides financières de l'OPATB parallèlement à la promulgation d'un nouvel arrêtés de ravalement obligatoire : arrêté n04-3645 du 10 avril 2007 en complément des arrêtés nº4-0613 du 5 mai 2004 et n04-3645 du 24 novembre 2004 (Carré, 2009).

30. In fine, la moitié des copropriétés ont bénéficié des aides Ademe qui étaient conditionnées à une consommation prévisionnelle inférieures à $110 \mathrm{kWh} / \mathrm{m}^{2}$.an.

31. On notera cependant que ce service a recruté spécialement un ingénieur thermicien pour suivre l'OPATB.

32. Les spécialistes du ravalement d'enduit maçonnés ont rapidement appris à intercaler une couche d'isolant thermique et ont concurrencé les pionniers qui s'étaient lancés sur le créneau 
de l'isolation par l'extérieur lorsque la ville de Grenoble a quasiment imposé cette isolation dans les constructions neuves (Henry, 2008).

33. Entretien de l'auteur avec deux entrepreneurs le 28 novembre 2009.

34. Délibération adoptée à l'unanimité du conseil de communauté le 25 septembre 2009.

35. Délibération du 25 septembre 2009 du conseil communautaire de Grenoble Alpes Métropole.

36. Précisément $5230 \mathrm{k} €$ de travaux et maitrise d'œuvre et $620 \mathrm{k} €$ d'animation pour une baisse de $575 \mathrm{tCO}_{2}$.

37. Précisément $620 \mathrm{k} €$ pour l'animation et $1580 \mathrm{k} €$ d'aides aux travaux pour une baisse de 575 $\mathrm{tCO}_{2}$.

\section{RÉSUMÉS}

Parmi les principaux gisements de réduction des émissions de gaz à effet de serre en France, la rénovation énergétique des bâtiments a fait l'objet d'un consensus lors du Grenelle de l'Environnement nonobstant son coût, estimé à plusieurs centaines de milliards d'euros. En l'absence d'obligations, difficiles à mettre en place juridiquement, les pouvoirs publics ont développé des dispositions incitatives. L'expérience des OPATB souligne le temps long de la filière constructive et l'intérêt d'un accompagnement public local, notamment pour les copropriétés. La généralisation passe par une fine articulation entre un dispositif local d'information et de financement et la fiscalité nationale. Ajuster un système multi-territorial conciliant les enjeux sociaux avec le facteur 4 nécessitera probablement quelques années d'expérimentations, d'évaluation, de débat et d'évolution des cadres national et locaux.

In France, the "Grenelle de l'Environnement" stakeholders agreed and planned on using thermal renovation on private buildings to reduce greenhouse gas emissions. They reached an agreement even though the cost was estimated as hundreds of billion euros. Its implementation is actually more difficult than expected: in the absence of the obligation to renovate, really hard to legally set up, public initiatives are required. The OPATB experience shows how slow this innovative process is and how important it is to support financially decision-makers and advise them especially for building co-owners. To locally articulate informative mission and support with a national tax fare system, requires agreements at a local and national level, which will probably take years to happen.

\section{INDEX}

Keywords : boundary object, building, co-ownership, France, greenhouse gas, insulation, RhôneAlpes, thermal refurbishment

Mots-clés : bâtiment, copropriétés, Echirolles, facteur 4, France, gaz à effet de serre, Grenoble, isolation thermique, objet-frontière, OPATB, réhabilitation 


\section{AUTEUR}

\section{GILLES DEBIZET}

Gilles Debizet est ingénieur en environnement (Grenoble-INP et ENPC). Il a piloté des projets de construction avant d'enseigner le génie civil et l'énergétique (agrégation ENS Cachan). Docteur en Aménagement de Paris-I en 2004, maître de conférences à l'Université Joseph Fourier depuis 2006, il enseigne le management environnemental et dirige la spécialité Maîtrise d'ouvrage et management de patrimoine bâti du master Sciences du Territoire de l'université de Grenoble. Chercheur au laboratoire PACTE, ses travaux portent sur la transformation de l'expertise urbaine, le management de projet et les interactions entre collectivités et acteurs de l'immobilier. Il intervient comme expert auprès de collectivités locales, de l'Ademe, de l'AERES et du ministère de la Culture. 